\title{
ESTADO DE LA INVESTIGACIÓN SOBRE EDUCACIÓN PARA \\ EL DESARROLLO SOSTENIBLE: UN ANÁLISIS \\ CIENCIOMÉTRICO DE LA PRODUCCIÓN CIENTÍFICA EN EL PERIODO 2005-2014
}

\author{
Juan David Sepúlveda Chaverra ${ }^{2}$
}

Recibido el 21 de julio de 2014, aprobado el 24 de febrero de 2015 y actualizado el 05 de mayo de 2015

DOI: 10.17151/luaz.2015.41.17

\section{RESUMEN}

La política nacional de educación ambiental en Colombia plantea la necesidad de tener modelos de soporte de la investigación ambiental; en este sentido, la identificación de tendencias, estructura y patrones dentro de la investigación mundial en Educación para el Desarrollo Sostenible -EDS- sirve de soporte para la formulación posterior de acciones que permitan cumplir con esta necesidad. Así, en este trabajo se propone la aplicación de un estudio cienciométrico de la producción de EDS, que permitió identificar componentes inherentes a la dinámica de la misma que la han llevado a alcanzar un nivel de crecimiento e interdisciplinariedad que la han llevado a un nivel de madurez como ciencia, impulsado principalmente a partir del impulso brindado por el Decenio de la Educación para el Desarrollo Sostenible de la UNESCO.

\section{PALABRAS CLAVE}

Cienciometría, educación para el desarrollo sostenible, sostenibilidad.

\section{STATUS OF RESEARCH ON EDUCATION FOR SUSTAINABLE DEVELOPMENT: A SCIENCE-METRIC STUDY OF SCIENTIFIC PRODUCTION IN THE PERIOD 2005-2014}

\begin{abstract}
National policy for environmental education in Colombia set out the need for environmental research supporting models. In this sense, identification of trends, structure and patterns inside global research in Education for Sustainable Development (ESD) serve as a support for the subsequent formulation of actions that would meet this need. Thus this work proposes the application of a science-metric study of the ESD production which allowed identifying components inherent to its dynamics that have led ESD to a level of maturity as a science driven mainly from the impulse given by the Decade of Education for Sustainable Development by UNESCO
\end{abstract}

\section{KEY WORDS}


Scence-metrics, education for sustainable development, sustainability.

\section{INTRODUCCIÓN}

En el periodo 2005-2014 se celebra el Decenio de las Naciones Unidas de la Educación para el Desarrollo Sostenible (EDS), y con este objetivo en mira se han adelantado múltiples acciones en diferentes ámbitos. En Colombia, a partir de la Política Nacional de Educación Ambiental, se ha hecho explícita la necesidad de contar con un modelo investigativo que sirva como componente fundamental de la investigación ambiental, donde el ambiente sea su objetivo y su campo de acción (Ministerio de Educación Nacional y Ministerio de Medio Ambiente, 2002), siendo primaria la necesidad de una investigación interdisciplinaria que brinde elementos y concepciones para la interpretación de las problemáticas contextuales.

El uso de técnicas y procesos de análisis cienciométrico permite la evaluación eficiente y objetiva del rendimiento de la investigación y la innovación, proveyendo una visión única de las fortalezas y debilidades de un campo de investigación (United Nations, 2007). Específicamente en la EDS, el uso de este tipo de análisis ha permitido revelar conocimiento sobre las tendencias de investigación latentes (Papadimitriou y Kidman, 2012) y además, como lo expresan Novo y Bautista (2012), ampliar el debate teórico y extender la información entre las partes interesadas en la EDS. De igual forma, este tipo de estudios sirve como soporte a la formulación posterior de estrategias de transferencia (Angulo et al., 2008) que favorecerían el desarrollo de modelos como los propuestos por el plan nacional de educación ambiental.

En este trabajo, a partir del estudio de las publicaciones científicas reseñadas en Scopus en el periodo 2005-2014 que abarca el Decenio de la EDS, se pretende identificar tendencias subyacentes a la producción científica relacionada con este campo.

\section{PLANTEAMIENTO METODOLÓGICO}

El propósito principal de este trabajo es abordar el análisis de la producción científica de mayor impacto relacionada con el estudio de la EDS y los campos donde esta opera, de manera que sea posible identificar características y tendencias que favorezcan posteriormente la formulación de acciones para el desarrollo de modelos investigativos acordes a los requerimientos y condiciones planteados en la política nacional de educación ambiental. 
En este sentido, se propone la aplicación de un estudio cienciométrico de la producción científica generada durante el Decenio de la EDS, buscando con ello identificar tendencias, patrones, lugares comunes y establecer las dimensiones vinculadas con la EDS dentro de la práctica investigativa a nivel mundial. El uso de la cienciometría en este proceso se basa en la necesidad de establecer tendencias y conocimiento específico sobre la dinámica de investigación en el campo de la EDS; la existencia de estudios precedentes (Novo y Bautista, 2012; Papadimitrou y Kidman, 2012) confirma la utilidad de esta herramienta de análisis en este campo particular.

Se seleccionaron 595 artículos de investigación reseñados en Scopus que corresponden con los siguientes criterios:

- Inclusión de: "education for sustainable development" dentro del título, el resumen o las palabras clave. El tema fue buscado de manera directa sin utilizar otros criterios o aproximaciones debido a que así es su denominación por parte de la UNESCO en el Decenio de la EDS.

- Artículos, trabajos en eventos y revisiones de tema. Se eliminaron del análisis 17 trabajos que corresponden a editoriales, reseñas de libros, capítulos de libro, debido a la poca profundidad investigativa de estos productos.

La selección de Scopus, aunque es clara la discusión sobre su importancia en la investigación debido a la baja participación de las ciencias sociales, permitió identificar un perfil de la EDS que vincula diferentes áreas y favorece el análisis de interdisciplinariedad y el nivel de inclusión de la EDS en otros campos temáticos. Las variables medidas corresponden con aquellas que permiten establecer el perfil y las características de la EDS a partir del análisis de las publicaciones; de igual forma, estas variables fueron seleccionadas por su utilización en estudios similares aplicados al mismo campo (Novo y Bautista, 2012; Papadimitrou y Kidman, 2012), con lo que es posible establecer algunos elementos comparativos contra conclusiones ya planteadas. A continuación la lista de variables utilizadas para el estudio:

- Productividad diacrónica.

- Curva de Price.

- Índice de Shannon-Weaver.

- Productividad geográfica.

- Productividad institucional.

- Productividad por autores.

- Productividad por áreas.

- Citaciones.

Igualmente, aplicando un proceso de análisis temático basado en el modelo de construcción de Angulo, Meriño y Sepúlveda (2007) se obtuvieron los mapas de correspondencias temáticos, que permiten establecer la estructura y analizar las principales tendencias conceptuales involucradas en la totalidad de la producción. 


\section{RESULTADOS Y ANÁLISIS}

\section{Perfil de las publicaciones}

El número de publicaciones es considerado como un indicador de actividad científica (United Nations, 2007) y, en este sentido, la productividad diacrónica es entendida como el número de artículos por cada año del periodo estudiado (Expósito y Fernández, 2012), y su estudio permite establecer la evolución de la productividad de un campo científico en el tiempo, además del grado de madurez del mismo, medido a partir de la ley de crecimiento exponencial de Price ${ }^{3}$. Para el caso de la EDS, la Figura 1 muestra el comportamiento de las publicaciones desde el año 1990, en el cual se encontró la primera referencia de acuerdo a los criterios de búsqueda.

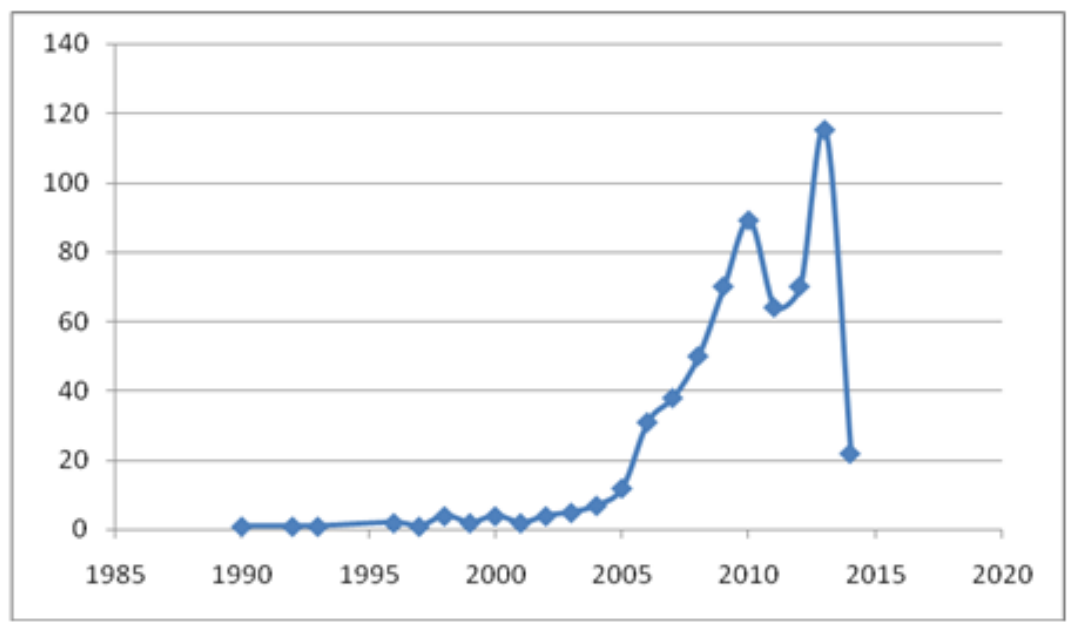

Fuente: Autor con datos extraídos de Scopus.

Figura 1. Evolución en el tiempo de publicaciones relacionadas con la EDS.

De acuerdo a la ley de crecimiento exponencial de Price, lo primero que se puede apreciar, es que la producción en el campo de la EDS toma la forma de una curva creciente, por lo que puede establecerse que se encuentra en la fase 2, esto da evidencia de un campo científico en proceso de maduración y, más importante aún, es posible verificar cómo su cota de crecimiento exponencial ha sido influida por el Decenio de la EDS iniciando su crecimiento en el año 2005, comprobando la importancia de este tipo de iniciativas sobre el desarrollo de nuevos campos científicos.

Ya en torno a la producción puntual de artículos en el periodo 2005-2014, se puede apreciar una tendencia creciente (la producción de 2014 incluye solo hasta el mes de marzo) con una notable disminución en los años 2011 y 2012, sin embargo un punto importante de este análisis es que muestra un escenario alternativo a la relación planteada por Novo y Bautista (2012) 
quienes en el análisis del caso español identificaron que la mayor productividad se encuentra asociada a la existencia de números especiales en las revistas científicas. Un caso que no se evidencia en el análisis de las ediciones y publicaciones estudiadas.

El perfil geográfico, institucional y de autores permite complementar la información obtenida sobre la evolución del campo con la identificación de los actores principales involucrados en la misma, datos que son útiles para identificar los líderes en el campo (Sepúlveda, 2011), además de identificar potenciales instituciones para el desarrollo de acciones colaborativas de investigación en el campo (Charris et al., 2010). En la tabla siguiente se puede apreciar la productividad y la participación de los 10 países líderes en producción académica sobre EDS.

Tabla 1. Países líderes en producción científica en EDS

\begin{tabular}{|l|c|c|}
\hline Pais & $\begin{array}{c}\text { No. } \\
\text { artículos }\end{array}$ & Participación \\
\hline United Kingdom & 89 & 0,14495114 \\
\hline Germany & 52 & 0,08469055 \\
\hline Australia & 44 & 0,07166124 \\
\hline United States & 41 & 0,06677524 \\
\hline Sweden & 39 & 0,06351792 \\
\hline Netherlands & 24 & 0,03908795 \\
\hline Canada & 23 & 0,03745928 \\
\hline Japan & 22 & 0,03583062 \\
\hline Greece & 18 & 0,02931596 \\
\hline Spain & 18 & 0,02931596 \\
\hline Otros & 244 & \\
\hline
\end{tabular}

Fuente: Elaboración propia con datos extraídos de Scopus.

Es así como el análisis de la productividad muestra investigaciones procedentes de Reino Unido que comprenden más del $14 \%$ del total de artículos publicados en este periodo con un total de 89 publicaciones, donde resaltan las universidades de Plymouth y Londres dentro de las 10 primeras instituciones en producción científica. Asimismo, dentro de las 10 primeras universidades de un total de 160 , que concentran el $20 \%$ de artículos se encuentran dos alemanas (Freie y Leuphana), dos australianas (Griffit y Royal Melbourne), una de Estados Unidos (Tennessee), dos suecas (Chalmers y Uppsala) y una holandesa (Delft). Con respecto a los autores, la Tabla 2 muestra la 
producción total de los cinco primeros junto con algunos datos bibliográficos.

Tabla 2. Principales autores en el campo de la EDS

\begin{tabular}{|c|c|c|c|c|c|c|}
\hline Autor & Nación & $\begin{array}{c}\text { No. } \\
\text { artículos }\end{array}$ & $\begin{array}{c}\text { No. } \\
\text { artículos } \\
\text { en } \\
\text { Scopus }\end{array}$ & Citaciones & $\begin{array}{c}\text { Colaboración } \\
\text { con otros } \\
\text { autores }\end{array}$ & Area \\
\hline Barth M. & Alemania & 8 & 13 & 121 & 16 & $\begin{array}{c}\text { Ciencias ambientales, } \\
\text { ciencias sociales }\end{array}$ \\
\hline Rieckmann M. & Alemania & 8 & 10 & 32 & 21 & $\begin{array}{c}\text { Ciencias ambientales, } \\
\text { energía }\end{array}$ \\
\hline Huisingh D. & $\begin{array}{c}\text { Estados } \\
\text { Unidos }\end{array}$ & 7 & 46 & 535 & 82 & $\begin{array}{c}\text { Ciencias ambientales, } \\
\text { energía }\end{array}$ \\
\hline Bogeholz S. & Alemania & 7 & 17 & 76 & 27 & Ciencias sociales \\
\hline Ferrer-Balas D. & España & 6 & 18 & 513 & 33 & $\begin{array}{c}\text { Ciencia de materiales, } \\
\text { ciencias sociales }\end{array}$ \\
\hline
\end{tabular}

Fuente: Elaboración propia con datos extraídos de Scopus.

\section{Publicaciones por área temática}

El ranking de publicaciones por tema permite apreciar las áreas particulares de investigación que reciben mayor atención por parte de la comunidad científica (Novo y Bautista, 2012), además, a partir de la estandarización de las áreas generales en los sistemas de indexación, el análisis de estas y la proporción en que representan la producción científica en un campo, es posible dar cuenta del enfoque interdisciplinario.

El análisis de las áreas de interés de los documentos analizados muestra un total de 21 campos de conocimiento cubiertos, donde las ciencias sociales son claramente dominantes representando el $44,3 \%$ de los temas cubiertos por las publicaciones. La Tabla 3 muestra la frecuencia acumulada de áreas declaradas en los trabajos analizados.

Tabla 3. Pareto de artículos analizados

\begin{tabular}{|l|c|c|c|}
\hline & Producción & Frecuencia & Acumulado \\
\hline Social Sciences & 407 & 0,44287269 & 0,44287269 \\
\hline Environmental Science & 156 & 0,16974973 & 0,61262242 \\
\hline Energy & 96 & 0,10446137 & 0,71708379 \\
\hline Engineering & 71 & 0,07725789 & 0,79434168 \\
\hline Business Management and Accounting & 60 & 0,06528836 & 0,85963003 \\
\hline Economics Econometrics and Finance & 21 & 0,02285092 & 0,88248096 \\
\hline Otros & 108 & 0,11751904 & 1 \\
\hline
\end{tabular}

Fuente: Elaboración propia con base en datos extraídos de Scopus. 
Aunque las ciencias sociales alcanzan casi la mitad de la producción, es importante resaltar cómo los temas se han relacionado en gran proporción con áreas como las ciencias ambientales, la energía, la economía y los negocios, siendo estas 6 áreas las que abarcan cerca del 90\% de la producción; en términos de las revistas, de las 10 primeras que concentran 230 publicaciones (el $50 \%$ de los artículos) 6 de ellas son especializadas en educación y las otras 4 son revistas especializadas en temas técnicos ambientales.

Ante este panorama, igualmente se aplicó la medida del índice de Shannon-Weaver para identificar el potencial de interdisciplinariedad asociado a los campos analizados, lo que arrojó como resultado que, a pesar de la alta concentración de ciencias sociales, el total de la producción en EDS evidencia un $61,8 \%$ de heterogeneidad en los temas, lo que indica que el campo ha alcanzado un alto grado de interdisciplinariedad en su producción.

\section{Estadísticas de citaciones}

Papadimitriou y Kidman (2012) al analizar las citaciones de publicaciones sobre educación ambiental y geográfica plantean que estas, aplicadas al campo de las ciencias sociales y humanidades, tienen como problema el bajo nivel de participación de publicaciones especializadas en este campo dentro de los principales sistemas de análisis y bases de datos. Al respecto Zitt y Bassecoulard (2008) plantean que las citaciones para estos campos miden la visibilidad mucho más que la calidad de las publicaciones, por lo que este indicador permite analizar la difusión de los resultados obtenidos dentro de una comunidad.

En esta sección, por tanto, se analizan no solo las estadísticas de citaciones de los trabajos para identificar tendencias en torno a la producción y su visibilidad, sino que también se evalúan las estadísticas de citaciones generales de las publicaciones en que estas aparecen, con la intención de identificar el potencial de visibilidad de los trabajos analizados.

Del universo de trabajos analizados un total de 313 concentran 2035 citaciones, cuyo comportamiento es relativamente similar, a la producción diacrónica, mostrando un retroceso en la cantidad de citaciones a partir del año 2011 tal como se puede observar en la figura siguiente. 


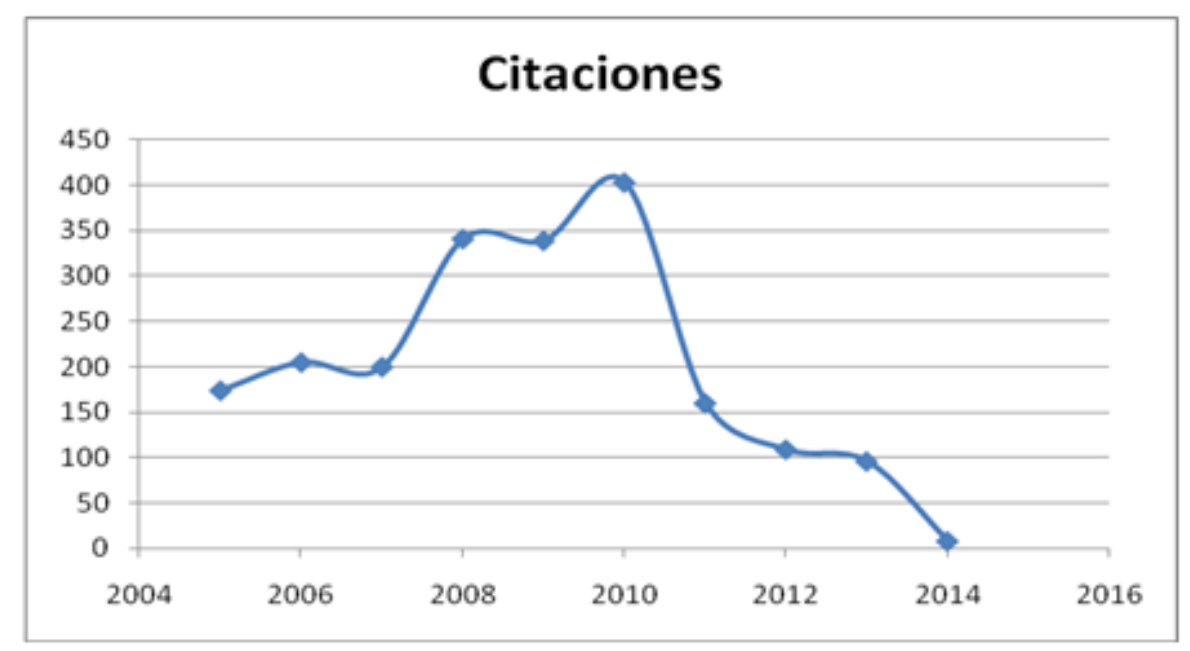

Fuente: Autor con datos extraídos de Scopus.

Figura 2. Evolución en el tiempo de las citaciones.

En el periodo analizado, la identificación de una tendencia de crecimiento y decrecimiento similar a la evolución de la producción diacrónica puede llevar a pensar que las citaciones corresponden exclusivamente a un modelo de endogenismo, donde los autores se citan entre ellos para aumentar los indicadores, sin embargo los datos existentes no son suficientes para soportar a esta hipótesis, y por el contrario, al analizar los datos se encuentra que el $20 \%$ de los trabajos (120 artículos) concentran el $80 \%$ de las citaciones, lo que da cuenta de un comportamiento normal en términos de la ley de Pareto. Esta práctica de acuerdo a la revista Información Tecnológica (2008) puede ser evidencia de "una muestra de la continuidad de un autor en su trabajo, que se trata de una subdisciplina nueva o muy especializada donde hay muy pocos documentos en la literatura", siendo esta última condición la más probable de acuerdo a los criterios de evolución del campo de la EDS ya analizados.

La tabla siguiente muestra los cinco primeros trabajos con mayor número de citaciones: 
Tabla 4. Autores con mayor número de citaciones

\begin{tabular}{|c|c|c|c|c|}
\hline Artículo & Autores & Año & Revista & No. citaciones \\
\hline $\begin{array}{l}\text { Globalization and } \\
\text { environmental education: } \\
\text { Lookingbeyond sustainable } \\
\text { development }\end{array}$ & Jickling, B., Wals, A. & 2008 & $\begin{array}{c}\text { Journal of } \\
\text { Curriculum } \\
\text { Studies }\end{array}$ & 57 \\
\hline $\begin{array}{l}\text { Deterring sustainability in } \\
\text { higher education institutions: } \\
\text { An appraisal of the factors } \\
\text { which influence sustainability } \\
\text { in higher education } \\
\text { institutions }\end{array}$ & $\begin{array}{c}\text { Velázquez, L., Munguia, N., } \\
\text { Sánchez, M. }\end{array}$ & 2005 & $\begin{array}{l}\text { International } \\
\text { Journal of } \\
\text { Sustainability } \\
\text { in Higher } \\
\text { Education }\end{array}$ & \\
\hline $\begin{array}{l}\text { Key competenciesin } \\
\text { sustainability: A reference } \\
\text { framework for academic } \\
\text { program development }\end{array}$ & $\begin{array}{l}\text { Wiek, A., Withycombe, L., } \\
\text { Redman, C. L. }\end{array}$ & 2011 & $\begin{array}{l}\text { Sustainability } \\
\text { Science }\end{array}$ & 50 \\
\hline $\begin{array}{l}\text { Dissonance in students' } \\
\text { perceptions of sustainable } \\
\text { development and } \\
\text { sustainability: Implications for } \\
\text { curriculum change }\end{array}$ & Kagawa, F. & 2007 & $\begin{array}{l}\text { International } \\
\text { Journal of } \\
\text { Sustainability } \\
\text { in Higher } \\
\text { Education }\end{array}$ & 45 \\
\hline $\begin{array}{l}\text { Embed ding sustainable } \\
\text { development at Cambridge } \\
\text { University Engineering } \\
\text { Department }\end{array}$ & $\begin{array}{c}\text { Fenner, R. A., Ainger, C. M., } \\
\text { Cruickshank, H. J., Guthrie, P. M. }\end{array}$ & 2005 & $\begin{array}{l}\text { International } \\
\text { Journal of } \\
\text { Sustainability } \\
\text { in Higher } \\
\text { Education }\end{array}$ & 42 \\
\hline
\end{tabular}

Fuente: Elaboración propia con base en datos extraídos de Scopus.

De igual forma, la figura siguiente muestra la distribución general de citaciones de las revistas donde se han presentado la mayor parte de los artículos ${ }^{4}$. 


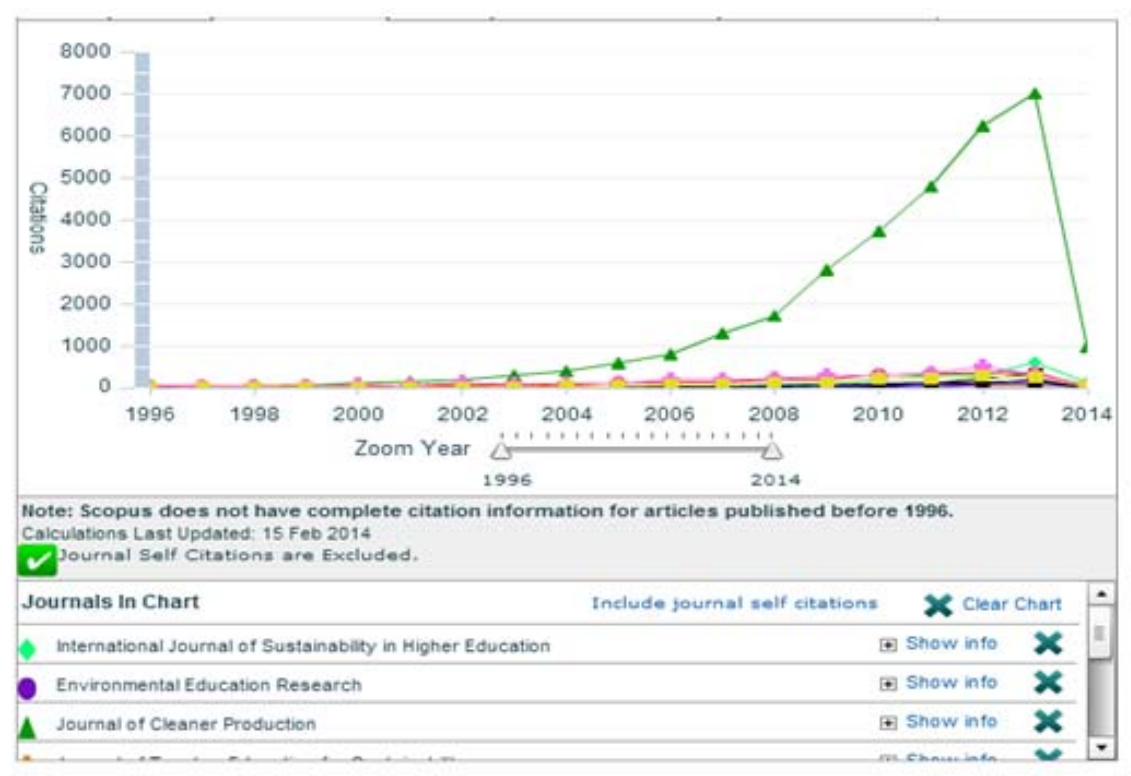

Fuente: Scopus.

Figura 3. Citaciones generales de revistas donde se publican los trabajos.

Tal como se puede apreciar, tres revistas destacan dentro de los mayores índices de citaciones a nivel general, en primer lugar, el International Journal of Sustainability in Higher Education donde se han presentado 50 de los artículos analizados. De igual forma, la revista Environmental Education Research que tiene 46 trabajos publicados en EDS, y la revista Journal of Cleaner Production con 38 artículos en el campo analizado.

Al respecto, cabe destacar la revista Journal of Cleaner Production, que en el periodo analizado creció de aproximadamente 500 citaciones a más de 7000 en el año 2013, igualmente en las publicaciones analizadas se observa una tendencia creciente en el número de citaciones obtenidas aunque no superan las 1000 citaciones por año. Estos resultados muestran un alto potencial de visibilidad de las publicaciones relacionadas con la EDS.

\section{Mapa de correspondencias temáticas}

Adicionalmente al análisis de los indicadores ya utilizados en estudios relacionados con el campo de la EDS, a este trabajo se integra el análisis de concurrencia temática (Angulo et al., 2007). El dendograma mostrado en la figura siguiente, fue obtenido mediante el análisis estadístico "N-Join" utilizando el paquete Ntsys y elaborado a partir de la conformación de una matriz de concurrencias de las palabras clave de los 539 artículos analizados $^{5}$. En esta figura se resumen los temas principales abordados por el universo de trabajos. 


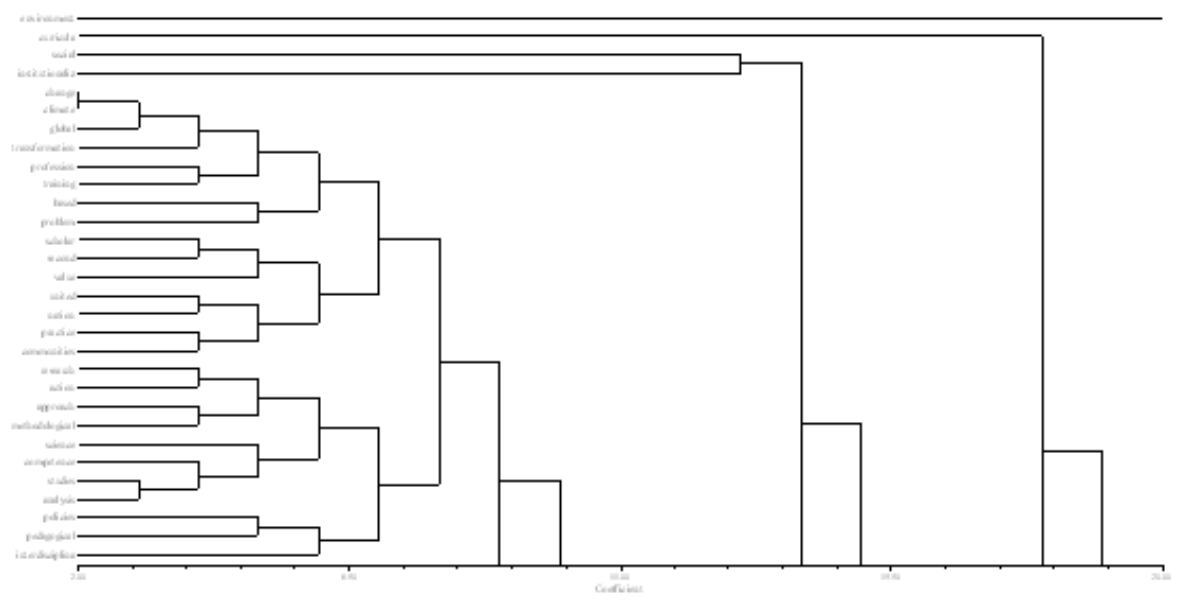

Fuente: Autor con datos extraídos de Scopus

Figura 4. Dendograma de correspondencia de temas.

A partir de la figura, es posible apreciar los siguientes clusters:

a) Cluster 1: compuesto por un conjunto de palabras clave que dan cuenta, en primer lugar, del problema del cambio climático (change y climate conforman la primera unión de conceptos en la totalidad del mapa) y el reto que este representa, siendo entonces necesaria la acción de la EDS a partir de la formación basada en problemas, valores y prácticas comunitarias. (Las palabras vinculadas a este cluster son: change, climate, global, transformation, profession, training, based, problem, scholar, second, value, united, nation, practice, communities).

b) Cluster 2: enfocado en el valor de la investigación como soporte a la práctica educativa, en este cluster los conceptos se vinculan alrededor de un perfil de vocabulario que integra la metodología, la práctica y los enfoques de la investigación científica con la interdisciplina, las competencias y las políticas orientadoras de la investigación con la pedagogía. (Las palabras vinculadas a este cluster son: research, action, approach, methodological, science, competence, studies, analysis, policies, pedagogical, interdiscipline).

Estos dos clusters, están al mismo tiempo vinculados por los siguientes conceptos: social, institution, curricula, environment. Esto último, dejando claro que la conformación de los currículos debe, en primer lugar, estar soportada en la dimensión educativa e investigativa $y$, al mismo tiempo, debe estar integrada a las condiciones y particularidades de las instituciones y el entorno social y ambiental de las mismas. 


\section{CONCLUSIONES}

La educación para el desarrollo sostenible se está configurando como un campo en maduración, con un crecimiento sostenido en el tiempo que le ha permitido alcanzar un alto grado de interdisciplinariedad en su producción, la cual además no está determinantemente ligada solo a la edición de números especiales en el tema, sino que además ya ha superado el umbral que determina el crecimiento natural de los campos científicos.

Estas características le han permitido superar la barrera de las ciencias sociales y la educación como el área dominante, siendo la EDS un campo científico que se está abriendo espacio en otros campos como las ciencias ambientales y la energía.

Aunque se logró analizar que no existe una relación de interdependencia en la EDS frente a números especiales de publicaciones, también fue posible establecer el impulso que políticas específicas le dan a los campos de conocimiento, en este caso particular, el impulso del Decenio de la EDS de la UNESCO, cuyo periodo significó la evolución de la EDS de un estado incipiente a un estado de madurez como campo científico

La orientación de la EDS vista desde las publicaciones analizadas muestra una orientación hacia una práctica pedagógica necesaria para enfrentar, a partir de la educación, los problemas sociales y ambientales en la dinámica del cambio global, al tiempo que debe estar soportada en un proceso formativo e investigativo acorde a las particularidades del entorno y la realidad de las instituciones.

\section{REFERENCIAS}

- Angulo, J., Meriño, L. y Sepúlveda, J. (2007). Metodología para la elaboración de mapas tecnológicos aplicado a celdas de combustible. Revista Teknos.

- Angulo, G., Meriño, L., Sepúlveda, J. y Charris, M. (2008). Diseño de un plan de transferencia de tecnologías en generación de energías para el departamento del Atlántico. Memorias I Congreso Internacional de Gestión Tecnológica e Innovación, Universidad Nacional, Bogotá.

- Bramardi, S. J. (2000). Estrategias para el análisis de datos en la caracterización de recursos fitogenéticos. Tesis de Doctorado. Departamento de Estadística e Investigación Operativa Universidad Politécnica de Valencia.

- Charris, M., Becerra, L., Angulo, G. y Camacho, J. (2010). Identificación de socios estratégicos: metodologías basadas en redes sociales e informetría. VIII Congreso Iberoamericano de Indicadores de Ciencia y Tecnología. España.

- Expósito, J. y Fernández, A. (2002). La productividad de la investigación sobre evaluación de programas educativos españoles 1975-2000. Revista de Investigación Educativa, 20(1), 
113-129.

Recuperado

de

http://revistas.um.es/rie/article/viewFile/97531/93591

- Información Tecnológica. (2008). Las autocitas en artículos de revistas de corriente principal. Información tecnológica, 19(5), 1.

Recuperado

http://www.scielo.cl/scielo.php?script=sci_arttext\&pid=S0718 $-07642008000$ $500001 \& \operatorname{lng}=e s \& t \mid n g=e s$

- Ministerio de Educación Nacional y Ministerio de Medio Ambiente. (2002). Política Nacional de Educación Ambiental SINA. Recuperado http://media.utp.edu.co/institutoambiental2011/archivos/proy ecto-ciudadano -de-educacionambiental/1421politicanacionaldeeducaiconambiental.pdf

- Novo, M. y Bautista, M. J. (2012). Análisis de la incidencia de la educación ambiental para el desarrollo sostenible en las revistas científicas españolas. Revista de educación, 358, 583-597.

- Papadimitriou, F. y Kidman, G. (2012). Statistical and scientometric analysis of international research in geographical and environmental education. Journal of International Research in Geographical and Environmental Education, 21(1), 11-20. Recuperado de http://eprints.qut.edu.au/56067/2/56067.pdf

- Sepúlveda, J. (2011). Diseño de un modelo y desarrollo de ejercicios de vigilancia para la toma de decisiones tecnológicas en el área de energías renovables, en la región de GuipúzcoaEspaña. Tesis de Maestría. Universidad del Magdalena.

- United Nations. (2007). Building science, technology and innovative systems for sustainable development in Africa. Recuperado de http://www.unep.org/training/programmes/Instructor\%20Versi on/Part_2/Activities/ Innovations_and_Technology/Environmentally_Sound_Techn ologies/Supplementall

Building Science and Technology Innovation Systems.pdf

- Zitt, M. y Basselcoulard, E. (2008). Challenges for scientometric indicators: data demining, knowledge flow measurements and diversity issues. Journal of Ethics in Science and Environmental Politics, 8, 49-60.

1. Investigación independiente dentro del programa de Doctorado en Desarrollo Sostenible de la Universidad de Manizales. Actualmente no presenta conflicto de interés.

2. Ing. MSc. Ph.D (c). Universidad de Manizales. juankarenium@gmail.com

3. http://www.ecured.cu/index.php/Bibliometr\%C3\%ADa\# Ley_de_crecimiento_exponencial

4. Esto es: el total de citaciones de las revistas y no solo citaciones relacionadas con estudios sobre la EDS.

5. Para el análisis de palabras clave, se establece que el $10 \%$ del vocabulario común al universo de documentos analizados representa de manera certera la estructura del mismo (Bramardi, 2000). 
Para citar este artículo: Sepúlveda Chaverra, J. D. (2015). Estado de la investigación sobre educación para el desarrollo sostenible: un análisis cienciométrico de la producción científica en el periodo 2005-2014. Revista Luna Azul, 41, 309-322. Recuperado de http://lunazul.ucaldas.edu.co/index.php?option=content\&tas $k=v i e w \& i d=1068$ 